\title{
Transcriptional activation of hedgehog target genes in Drosophila is mediated directly by the Cubitus interruptus protein, a member of the GLI family of zinc finger DNA-binding proteins
}

\author{
Cyrille Alexandre, Antonio Jacinto, and Philip W. Ingham ${ }^{1}$ \\ Molecular Embryology Laboratory, Imperial Cancer Research Fund, London, WC2A 3PX, United Kingdom
}

\begin{abstract}
Members of the Hedgehog (Hh) family of secreted proteins have been identified recently as key signaling molecules that regulate a variety of inductive interactions central to the development of both Drosophila and vertebrates. Despite their widespread importance, the way in which Hh signals are transduced inside the cell remains poorly understood. The best candidate for a transcription factor that mediates $\mathrm{Hh}$ signaling in Drosophila is the product of the cubitus interruptus (ci) gene, a zinc finger protein that exhibits significant homology to protein products of the vertebrate GLI gene family. Here, we show that elevated levels of Ci are sufficient to activate patched (ptc) and other $h h$ target genes, even in the absence of $h h$ activity. We also show that $\mathrm{Ci}$ can function as a transcriptional activator in yeast and demonstrate that the zinc finger domain of the protein is sufficient for its target specificity. Finally, we identify sequences in the promoter region of the ptc gene, a primary target of $\mathrm{Hh}$ signaling, that are identical to the consensus-binding sequence of the GLI protein and are required for reporter gene expression in response to Hh activity. Taken together, our results strongly support the role for $\mathrm{Ci}$ as the transcriptional activator that mediates $h \boldsymbol{h}$ signaling.
\end{abstract}

[Key Words: hedgehog signaling; patched promoter; GLI family; cubitus interruptus; decapentaplegic; wingless]

Received May 5, 1996; revised version accepted July 2, 1996.

Molecular mechanisms that control pattern formation in many developmental systems have been shown to involve key signaling molecules such as members of the Hedgehog family of secreted proteins. Of particular importance in vertebrates is the Sonic hedgehog (Shh) protein, the activity of which has been implicated in the patterning of the developing limb (Riddle et al. 1993), the neural tube and somites (Echelard et al. 1993; Krauss et al. 1993; Johnson et al. 1994; Roelink et al. 1994, 1995; Fan et al. 1995), as well as in gut morphogenesis (Roberts et al. 1995) and left-right asymmetry (Levin et al. 1995). In addition, other members of the family play important roles in chondrocyte differentiation (Vortkamp et al. 1996), spermatogenesis (Bitgood et al. 1996), and muscle cell specification (Currie and Ingham 1996).

\footnotetext{
${ }^{1}$ Corresponding author.
}

In Drosophila, hedgehog (hh) was first identified as a member of the segment polarity class of genes (NüssleinVolhard and Wieschaus 1980), which function collectively to organize the patterning of both the embryonic segments and imaginal discs. Various lines of evidence suggest that the role of $h h$ in this process is primarily to regulate the expression of other signal encoding genes. In the embryo, the principal target of hh activity is another segment polarity gene wingless (wg), which like $h$ h encodes a secreted protein (Rijsewijk et al. 1987; van den Heuvel et al. 1989). Transcription of $w g$ is maintained just anterior to each parasegment boundary by the activity of $h h$ in neighboring cells (Hidalgo and Ingham 1990; Ingham 1993), thus ensuring a discrete source of the Wg organizing signal in each segmental field. In the imaginal discs, $h h$ performs a similar role, establishing sources of the organizing activities encoded by wg (Couso et al. 1993; Struhl and Basler 1993/ and decapentaplegic $\langle d p p|$ (Nellen et al. 1996) by promoting the transcription of both genes along the anteroposterior compartment boundary (Basler and Struhl 1994; Capdev- 
ila and Guerrero 1994; Tabata and Kornberg 1994; Ingham and Fietz 1995).

Although the patterning activities of $\mathrm{Hh}$ molecules are well known, their signal transduction pathways remain unclear. Most of what is known derives from genetic analysis in Drosophila, where a number of genes belonging to the segment polarity class have been implicated in hh signal transduction (Ingham et al. 1991; Forbes et al. 1993; Ingham 1993; Motzny and Holmgren 1995). Among these genes is cubitus interruptus (ci), loss-offunction mutations of which are embryonic lethal and have phenotypes similar to those of $h h$ (Orenic et al. 1987). Transcription of $w g$ disappears from early stage 10 onward in ci mutant embryos (Hidalgo 1991; Forbes et al. 1993; Hooper 1994; Slusarski et al. 1995), even in the presence of high levels of ubiquitous $h h$ expression $(M$. Fietz and P. Ingham, unpubl.), strongly suggesting that $c i$ is required for the $h h$-dependent regulation of $w g$ transcription. Mutations of $c i$ also have effects on the patterning of imaginal structures, the gene name deriving from the interrupted fifth or cubitus vein of the wing caused by certain dominant gain-of-function alleles of the locus (Stern and Kodani 1955). Although the basis of this phenotype is unclear, phenotypes associated with a reduction of ci activity are similar to those caused by mutations of fused (Fausto-Sterling 1978; Slusarski et al. 1995), another segment polarity gene implicated in $h h$ signaling (Forbes et al. 1993; Ingham 1993; Thérand et al. 1996).

The ci gene encodes a protein with zinc fingers, highly homologous to protein products of the vertebrate GLI gene family and the Caenorhabditis elegans sex determination gene tra-1 (Orenic et al. 1990; Zarkower and Hodgkin 1992). The GLI and tra-1 proteins bind DNA in a sequence-specific manner (Kinzler and Vogelstein 1990; Zarkower and Hodgkin 1993) and the crystal structure of the GLI-DNA-binding complex has been solved (Pavletich and Pabo 1993). Thus, the Ci protein seems to be a good candidate for the transcription factor that mediates the activation of gene expression in response to $h h$ signaling. The distribution of Ci protein in both embryos and imaginal discs is consistent with such a role, being expressed in a pattern complementary to that of $h h$ transcription (Johnson et al. 1995; Motzny and Holmgren 1995). Thus, $\mathrm{Ci}$ is present in cells that respond to $\mathrm{Hh}$ by transcribing wg or $d p p$, as well as in cells that normally express neither gene but are capable of responding to ectopic $h$ h activity (Ingham 1993; Basler and Struhl 1994). This distribution suggests that the protein is present in most cells in an inactive form, its activation occurring in direct response to cells receiving the $\mathrm{Hh}$ signal. In line with this, there is a significant increase in levels of $\mathrm{Ci}$ protein, but not $c i$ transcript, in cells responding to Hh (Motzny and Holmgren 1995) or in cells in which the Hh pathway is activated constitutively through the removal of protein kinase A activity (Johnson et al. 1995), indicating that $h h$ regulates $c i$ expression post-transcriptionally. It is, however, notable that the vast majority of $\mathrm{Ci}$ protein is localized in the cytoplasm irrespective of whether cells are responding to
Hh (Motzny and Holmgren 1995). Thus, Hh does not seem to direct nuclear uptake of Ci but instead seems to act by increasing the overall levels of $\mathrm{Ci}$ within the cell.

In this study we have investigated the relationship between $\mathrm{Ci}$ and Hh signaling by using the GAL4 UAS (upstream activating sequences) system (Brand and Perrimon 1993) to direct high-level ectopic expression of the protein both in the embryo and in imaginal discs. We find that elevated levels of $\mathrm{Ci}$ are sufficient to activate $h h$ target genes, even in the absence of hh activity. We also show that $\mathrm{Ci}$ activates transcription in yeast by a GLI consensus-binding site and demonstrate that the zinc finger domain is sufficient for its target specificity. Finally, we identify several consensus-binding sites of the GLI protein in the patched (ptc) promoter region, a primary target of Hh signaling, and demonstrate that these sequences are required for activation of $p t c$ transcription in response to $\mathrm{Hh}$ activity. Our results strongly support a role for $\mathrm{Ci}$ as the transcriptional activator that mediates $h h$ signaling and provide the first direct evidence that $c i$ performs this function in the imaginal discs as well as in the embryo.

\section{Results}

Misexpression of Ci activates wg and ptc transcription in the embryo

During normal embryogenesis the activity of $h h$ in the engrailed (en) expressing cells at the anterior boundary of each parasegment maintains the transcription of the wg gene in cells immediately anterior and adjacent to those expressing en (Fig. 1A,B; Fig. 2A,B) and of ptc in two stripes of cells flanking each en domain (Fig. 1G,H). Expression of both genes similarly depends on ci activity, suggesting that $c i$ mediates $h h$ activity (Forbes et al. 1993). To determine whether $c i$ activity is sufficient to activate transcription of $w g$ and ptc, we used the GAL4 system to misexpress $\mathrm{ci}$ in cells where the gene is not normally transcribed. Flies carrying the $c i$ coding region cloned downstream of yeast upstream activating sequences (UASci) were crossed to enGAL4 flies (see Fietz et al. 1995). Embryos derived from this cross die at the end of embryogenesis and express high levels of $\mathrm{Ci}$ protein within the en domains (data not shown). The expression domains of both $\mathrm{wg}$ and $p t c$ in such embryos are significantly broader than in their wild-type sibs, suggesting that both genes are activated by the ectopically expressed Ci protein (Fig. IC,D and I,J). To confirm this, the broadened expression domains were related to the en domains using double labeling techniques. In both cases, the broadening can be seen to be attributable to the ectopic activation of either gene in the en-expressing cells (Fig. 2C-F). Thus, expression of $c i$ is sufficient to activate wg and $p t c$ transcription.

We then asked whether activation of $w g$ and $p t c$ transcription depends on the levels of Ci protein within a cell. To do this we used the ptcGAL4 line (Speicher et al. 1994) to express Ci at high levels throughout the normal ci expression domain. Such high-level expression results in the ectopic activation of both $w g$ and ptc (Fig. 1E,F and 

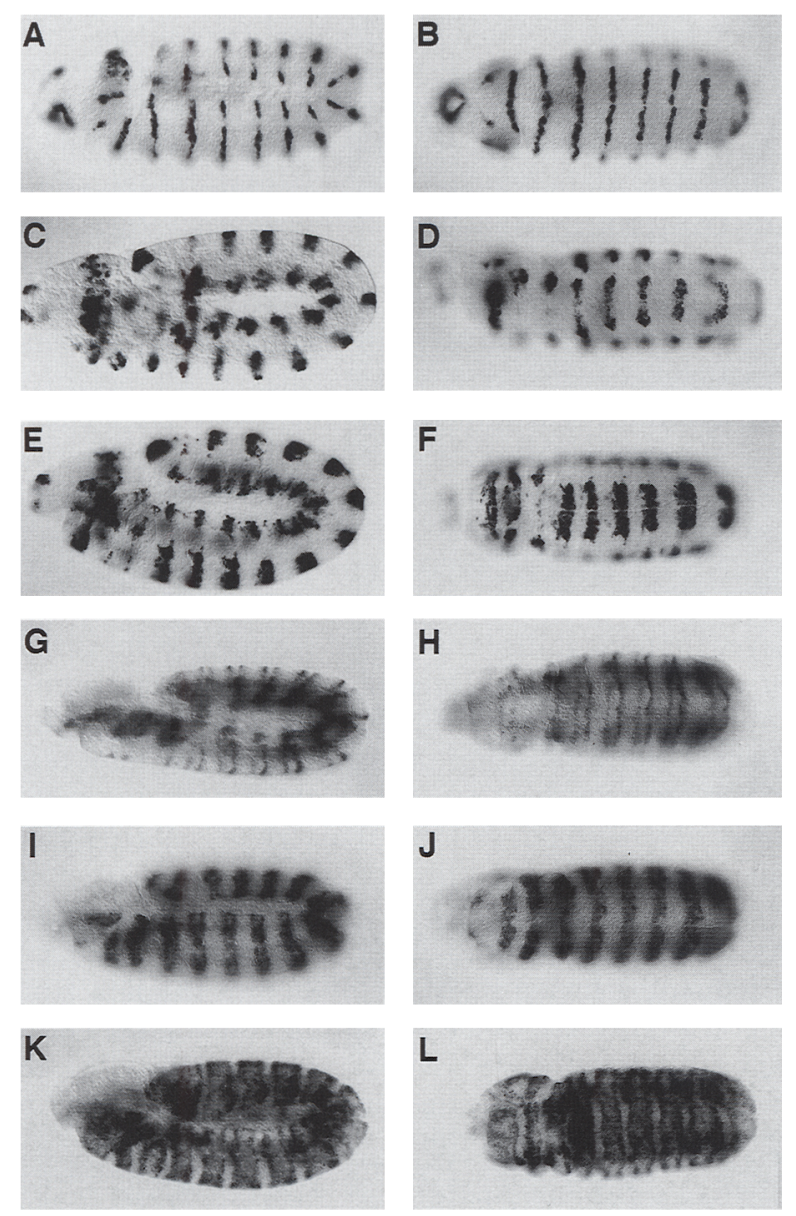

Figure 1. Ectopic transcription of wg and ptc induced by misexpression of $\mathrm{Ci}$ during embryogenesis. (Right) lateral view; (left) ventral view. Distribution of $w g$ transcripts in wild type $(A, B)$, enGAL4 $/+$; UASci $/+(C, D)$ and ptcGAL4 $/+$, UASci $/+$ $(E, F)$ embryos at stage 11 . Distribution of $p t c$ transcripts in wild type $(G, H)$, enGAL4/+; UASci $+(I, I)$ and ptcGAL4/ +; UAScil $+(K, L)$ embryos at stage 11 . In each case, ectopic $\mathrm{Ci}$ activity results in the broadening of both $w g$ and ptc expression domains $(C-F)$ and $(I-L)$; note, however, the restricted expansion of $w g$ compared to that of ptc.

$\mathrm{K}, \mathrm{L}$ ) in a manner similar to that caused by ubiquitous expression of hh (Ingham 1993; Tabata and Kornberg 1994). Thus, ptc is expressed at uniformly high levels between each en domain, whereas wg transcription expands anteriorly to occupy most or all of the cells competent to express wg (Ingham et al. 1991), as defined by the expression of sloppy paired (slp) (Grossniklaus et al. 1992; Cadigan et al. 1994).

High-level expression of $\mathrm{Ci}$ is sufficient to activate $\mathrm{wg}$ transcription in the absence of hh activity

Absence of $h$ h activity results in the loss of $w g$ transcription and concomitantly in the loss of anteroposterior polarity in the larval segments, the posterior naked cuticle being eliminated from every segment. To determine whether high-level expression of $c i$ is sufficient to activate $w g$ transcription even in the absence of $h h$ activity, we used a hairyGAL4 line (Brand and Perrimon 1993) to misexpress $c i$ in $h$ h mutant embryos (see Materials and methods) (Fig. 3). Because hairy (h) is expressed only in alternating parasegments (Ingham et al. 1985), expression of $c i$ under $h$ control should rescue only alternate stripes of wg expression. Consistent with this, the cuticles of UASci; $h$ GAL $4 h h^{I f}$ embryos exhibit a pair-rule phenotype, where every other segment now shows naked cuticle, normally indicating $\mathrm{Wg}$ activity (Fig. 3B). In situ hybridization reveals that the even-numbered stripes of $w g$ expression are indeed present in such embryos; in addition, the odd-numbered stripes 9,11 , and 13 are also usually rescued and the more posterior even-numbered stripes are expanded (Fig. 3D). We interpret these latter effects as being attributable to the temporal difference in the resolution of the $h$ pattern along the anteroposterior axis, the more posterior stripes extending over a width greater than one segment and hence activating $w g$ in adjacent parasegments.
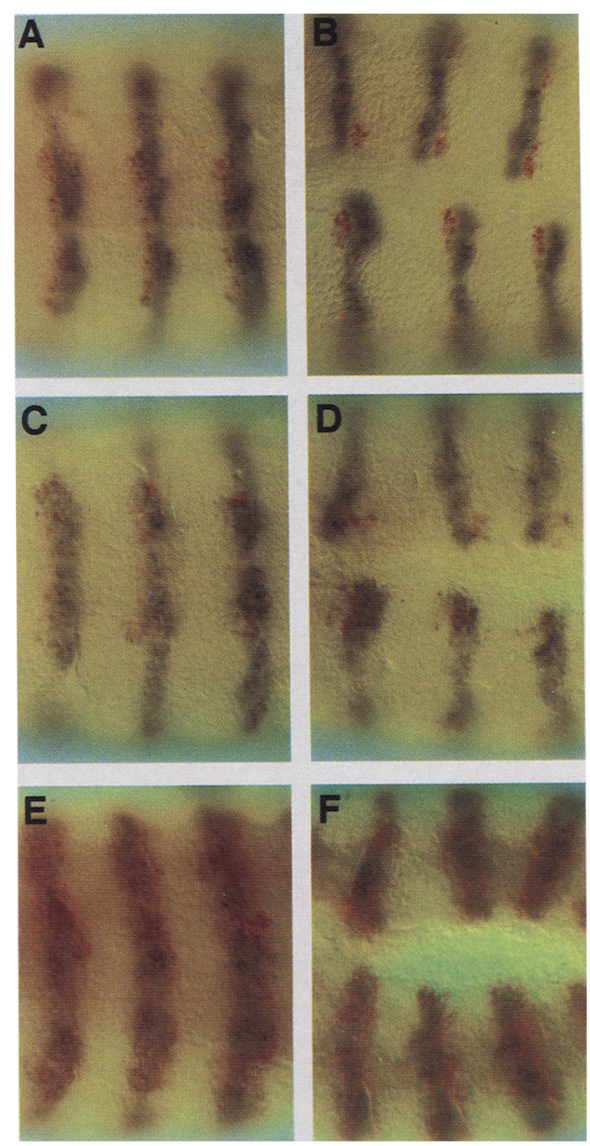

Figure 2. Transcription patterns of en (dark brown), wg (red) $(A-D)$, and $p t c$ (red) $(E, F)$ in embryos misexpressing Ci. (Right) ventral view; (left $)$ lateral view. $(A, B)$ In wild-type embryos, wgexpressing cells are immediately anterior and adjacent to the en domain. In embryos misexpressing $\mathrm{Ci}$ in the en domain, transcription of $w g(C, D)$ and $p t c(E, F)$ is strongly activated by $C i$ in en-expressing cells. 


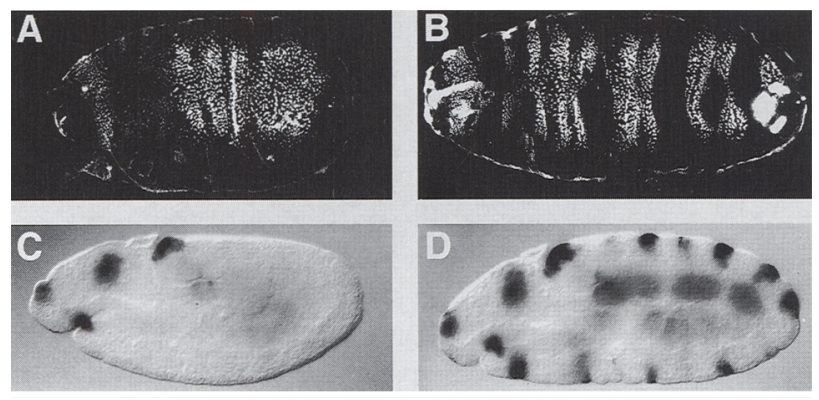

Figure 3. Rescue of $h h$ mutant embryos by misexpression of $c i$. (A) Ventral cuticle pattern of a pharate larva homozygous for $h h^{\prime \prime}$; the absence of naked posterior cuticle is presaged by the loss of $w g$ expression in the stage 10 embryo $(C)$. (B) Ventral cuticle pattern of a $h h^{\prime \prime}$ homozygote expressing $\mathrm{Ci}$ under the control of the hairy enhancer elements. Note the rescue of posterior naked cuticle in alternate segments and the restoration of $w g$ expression in alternate parasegments $(D)$.

\section{High-level misexpression of ci activates hh targets in} imaginal discs

As in the embryo, $c i$ is expressed in imaginal discs in a pattern reciprocal to that of $h h$. Thus, in the wing disc, $\mathrm{Ci}$ protein is restricted to the anterior compartment with elevated levels accumulating just anterior to the compartment boundary (Johnson et al. 1995; Motzny and Holmgren 1995), a region where transcription of ptc and $d p p$ is activated in response to $h h$ signaling (Basler and Struhl 1994; Capdevila and Guerrero 1994; Ingham and Fietz 1995). To investigate whether high levels of Ci can activate transcription of these $h h$ target genes in the disc, we used the GAL4 line 30A (Brand and Perrimon 1993) to drive expression of $\mathrm{Ci}$ in a ring around the presumptive wing blade (Fig. 4). Such 30A;UASci animals die during late larval or pupal stages; pharate adults exhibit outgrowths from the proximal wing region similar to those caused by ectopic $h h$ or $d p p$ expression driven by the same GAL4 line (data not shown) (Capdevila and Guerrero 1994; Ingham and Fietz 1995). The effects of ectopic $\mathrm{Ci}$ (Fig. 4A,D) on $d p p$ and $p t c$ transcription were assayed using the $d p p-l a c Z$ reporter gene construct \{Blackman et al. 1991) and the $p t c-1 a c Z$ enhancer trap line H84. In the third larval instar disc, expression of $d p p-l a c Z$ is activated ectopically in all cells expressing high levels of $\mathrm{Ci}$ in the anterior compartment, but is not activated in the posterior compartment (Fig. 4B,C). Expression of the ptc-lac $Z$ enhancer trap, in contrast, is activated ectopically throughout the $30 \mathrm{~A}$ expression domain in both compartments (Fig. 4E,F). Thus, as in the embryo, high levels of $\mathrm{Ci}$ protein are sufficient to activate transcription of $p t c$, even in the presence of En; however, ectopic $\mathrm{Ci}$ activity apparently cannot overcome the repression of $d p p$ transcription by En (Sanicola et al. 1995).

\section{Functional domains of Ci protein}

The $\mathrm{Ci}$ protein contains at least three distinct domains: (1) an amino-terminal region rich in alanine, a character- istic of repression domains in some Drosophila transcriptional repressors, [e.g., Engrailed (En) (Han and Manley 1993) and Krüppel (Kr) (Licht et al. 1990)]; (2) a zinc finger domain that shows a high degree of homology with the DNA-binding zinc finger domain of the vertebrate GLI proteins (84-93\%) (Kinzler and Vogelstein 1990; Ruppert et al. 1990); and (3) a carboxy-terminal domain that is highly acidic, a property typical of activation domains in transcription factors (Mitchell and Tiian 1989|.

To determine which regions of the $\mathrm{Ci}$ protein are necessary for the regulation of $h h$ target gene transcription by $\mathrm{Ci}$, we generated a number of constructs in which these different domains are deleted or replaced by heterologous domains (Fig. 5). The modified constructs were cloned downstream of UAS, transformed into flies, and assayed for their ability to activate $p t c$ and $d p p$ transcription in imaginal discs. Deletion of the amino-terminal alanine-rich domain has no effect on the ability of the protein to activate either $p t c$ or $d p p$ transcription (Fig. $6 \mathrm{~B}, \mathrm{E})$. In contrast, deletion of the carboxy-terminal acidic domain abolishes $p t c$ and $d p p$ transcriptional activation (data not shown). We then generated a construct in which most sequences both amino-terminal and carboxy-terminal to the zinc finger domain are deleted and the remaining region fused to the herpes simplex virus (HSV)VP16 transcriptional activation domain (see Fig. 5) (Triezenberg et al. 1988). This construct, ZFCiVP16, retains the ability to activate both $p t c$ and $d p p$ transcrip-
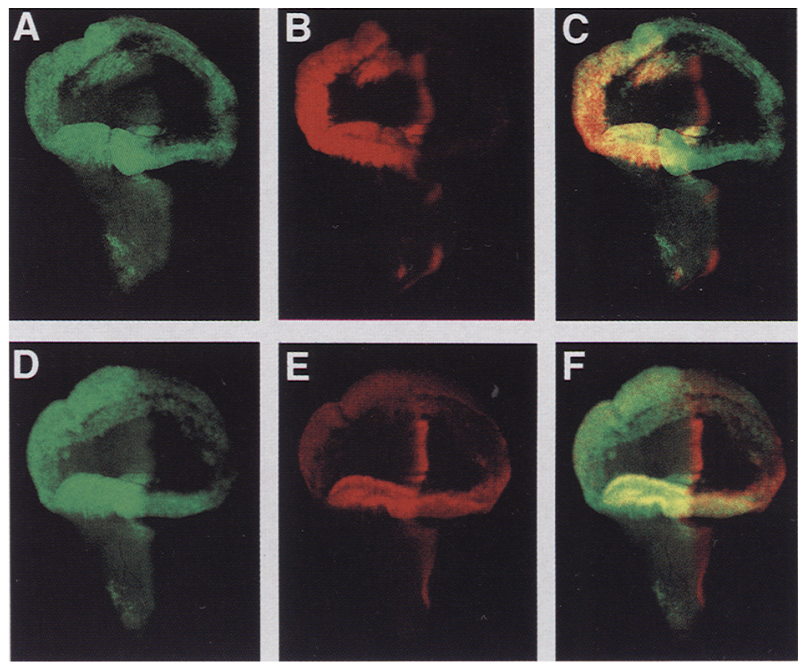

Figure 4. Ectopic induction of $d p p$ - and $p t c$ - reporter genes by misexpression of $\mathrm{Ci}$ in wing imaginal discs. Anterior is to the left in all cases. $\{A, D\}$ Ectopic expression of $\mathrm{Ci}$ (green) in a ring around the presumptive wing blade driven by the GAL4 line 30A. The levels of ectopic protein are much higher than those generated in the anterior compartment by the endogenous gene. Ectopic expression of $d p p$ (red) is only present in the anterior compartment $(B)$; whereas the ectopic expression of ptc (red) overlaps the misexpressed $\mathrm{Ci}$ protein in both compartments $\langle E\rangle$. $(C, F)$ Superimposition of the individual stainings showing a precise coincidence between $\mathrm{Ci}$ and the reporter genes. 


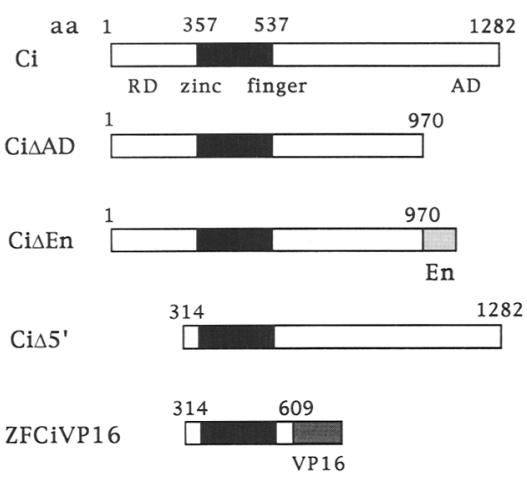

Figure 5. Schematic representation of $\mathrm{Ci}$ deletion and fusion constructs containing the transcriptional repression domain from En (amino acids 228-282) or the transcriptional activation domain from (HSV)VP16 (amino acids 418-490). The putative $\mathrm{Ci}$ repressor domain (RD) is localized $5^{\prime}$ to the zinc finger region, whereas the putative activation domain $(\mathrm{AD})$ is localized at the carboxyl terminus of the $\mathrm{Ci}$ molecule.

tion in the wing disc, although the induction is not as robust as that achieved with the full-length protein or the amino-terminal deletion (Fig. $6 \mathrm{C}, \mathrm{F}$ ); this may indicate that regions outside the zinc finger domain contribute to the stability or activity of the protein.

Finally, we generated a construct in which the putative activation domain is replaced with the repression domain of the En protein (see Fig. 5) (Han and Manley 1993; Badiani et al. 1994). Expression of this construct in the presumptive wing pouch fails to activate $d p p$ transcription; instead it leads to the repression of the normal
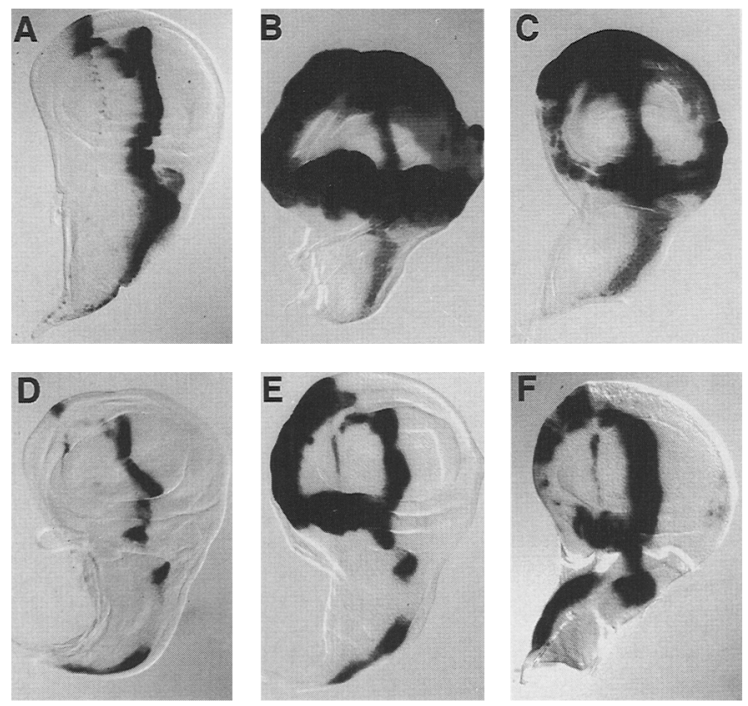

Figure 6. Functional domains of the $\mathrm{Ci}$ protein. Normal expression of $p t c-l a c Z(A)$ and $d p p-l a c Z(D)$ in the wing imaginal disc. Ectopic expression of $p t c-l a c Z(B)$ and $d p p-l a c Z(E)$ where a truncated form of $\mathrm{Ci}\left(\mathrm{Ci} \Delta 5^{\prime}\right)$ (see Fig. 5) is expressed ectopically using the 30A GAL4 line. The zinc finger of Ci fused to the activation domain of VP16 (ZFCiVP16) gives similar effects $(C, F)$. transcription of $d p p$ (Fig. 7D) and ptc (data not shown) along the anteroposterior compartment boundary, as would be expected if the domain substitution has converted the protein from a transcriptional activator to a repressor. Interestingly, the wings differentiated by such animals are characterized by a fusion between vein L3 and L4 (Fig. 7C); similar effects are induced by the overexpression of Ptc, which suppresses the hh signaling pathway, using the same GAL4 driver line (Fig. 7E,F) (Johnson et al. 1995).

\section{Ci can activate transcription in yeast}

The functional dissection of the Ci protein described above strongly suggests but does not prove that $\mathrm{Ci}$ acts as a direct regulator of transcription. To demonstrate this unequivocally we generated a yeast strain containing a HIS 3 reporter gene under the control of one GLI consensus-binding site (Kinzler and Vogelstein 1990) and tested the ability of $\mathrm{Ci}$ to activate transcription from this construct in a heterologous system, Saccharomyces cerevisiae. Yeast cells were cotransformed with plasmids containing the HIS 3 reporter gene and the ci coding region cloned downstream of the galactose-inducible promoter. Control and cotransformed cells were tested for their ability to grow on yeast medium lacking histidine in the presence or absence of galactose; only cells carrying both plasmids grew on the selective medium and then only in
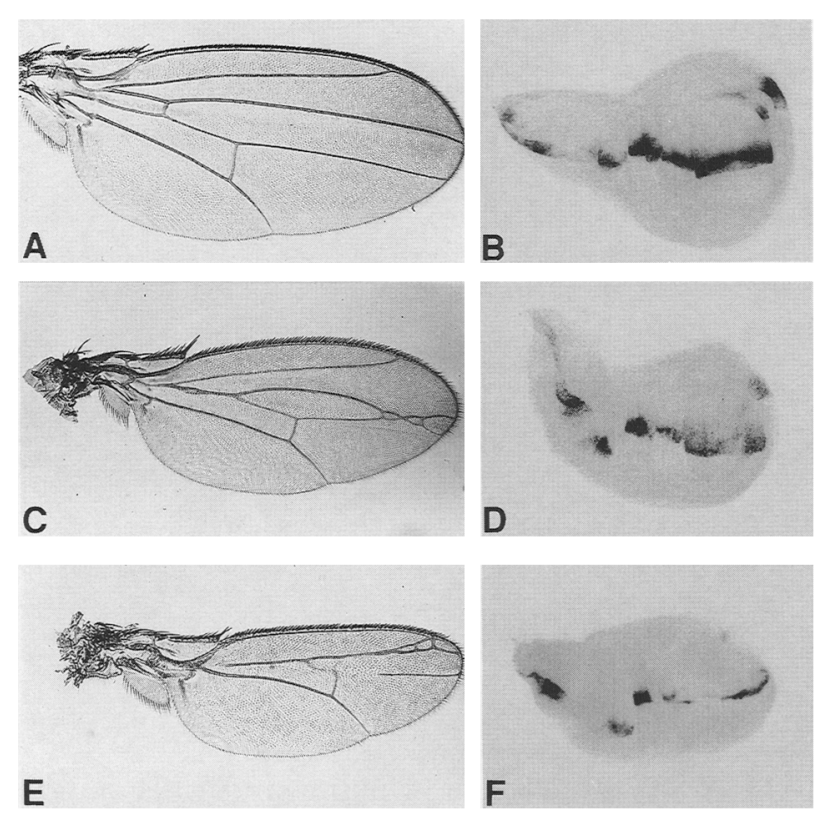

Figure 7. Repression of $d p p$ transcription by a repressor form of $\mathrm{Ci}(\mathrm{Ci} \Delta \mathrm{En})$. Wild-type Drosophila wing showing the normal distance between $\mathrm{L} 3$ and $\mathrm{L} 4$ veins $(A)$ and $d p p-l a c Z$ expression in the wing imaginal disc $(B)$. Wing patterning defects $(C, E)$ and repression of $d p p$ transcription in the wing imaginal disc $(D, F)$ caused by the overexpression of Ci $\Delta \mathrm{En}$ and by wild-type Ptc protein, respectively, using the $71 \mathrm{~B}$ GAL4 enhancer line. Note the fusion of vein L3 and L4 and the reduction in wing blade size. 
the presence of galactose (Fig. 8), indicating that the $\mathrm{Ci}$ protein can induce transcription from the synthetic promoter containing the GLI-binding site. Moreover, this activation is specific to the GLI-binding site as $\mathrm{Ci}$ is unable to activate transcription from the same HIS3 reporter gene under the control of the yeast transcription factor MCM1-binding site (STE3 UAS; Bender and Sprague 1987).

\section{Sequences upstream of ptc that include GLI-binding} sites drive transcription in response to Hh activity

Previous studies have shown that sequences from -2000 to +500 of the ptc transcription unit are sufficient to drive reporter gene expression in cells adjacent to the $h h$ domain in both embryos and imaginal discs, indicating that they contain elements responsive to the hh signal (Forbes 1992; Forbes et al. 1993). Sequence analysis of this fragment reveals the existence of a cluster of three GLI consensus-binding sites centered at position -684 relative to the transcription start site (Fig. 9). Deletion of sequences distal to these binding sites (FE construct; Fig. 9) leaves the compartment boundary-specific expression of reporter gene intact (Fig. 10A). Moreover, expression of this reporter construct can be induced ectopically by shh activity driven by the GAL4 line 30A (Fig. 10C), indicating that FE still retains the $h h$ responsive elements. Deletion of a further $156 \mathrm{bp}$, however, which includes all three GLI consensus sites (GE construct; Fig. 9|, abolishes both the robust compartment boundary-specific expression and the shh inducibility (Fig. 10B,D). Therefore, we conclude that sequences
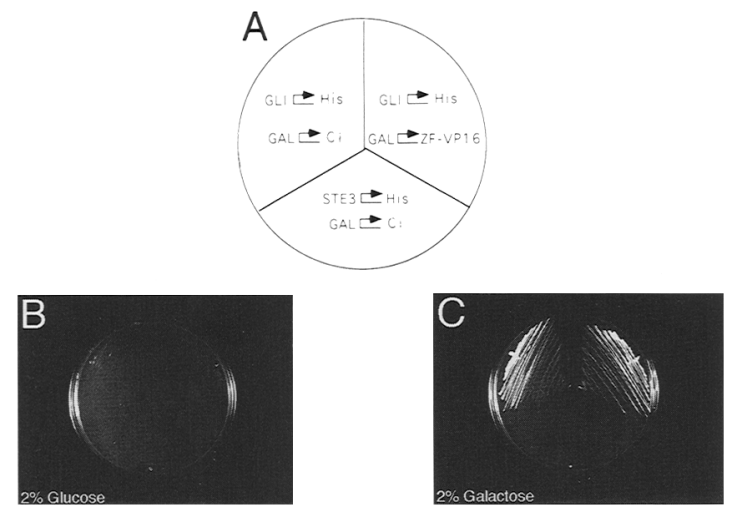

Figure 8. Ci activates transcription specifically through a GLI consensus-binding site in an heterologous system (S. cerevisiae). (A) Panel showing the three different cotransformations: The full-length $\mathrm{Ci}$ and the zinc finger region fused to VP16 activation domain are under the control of the galactose-inducible promoter. Note the activation of the HIS 3 reporter gene by these two different $\mathrm{Ci}$ constructs when they are cotransformed with the reporter gene under the control of one GLI consensusbinding site and streaked on $\mathrm{His}^{-}, 2 \%$ galactose plate $\langle C|$, but no growth is detected in the presence of $2 \%$ glucose $(B)$. A cotransformation of the HIS 3 reporter gene under the control of the MCMl-binding site and the $\mathrm{Ci}$ expression vector does not permit growth on $\mathrm{His}^{-}, 2 \%$ galactose plate $(C)$.

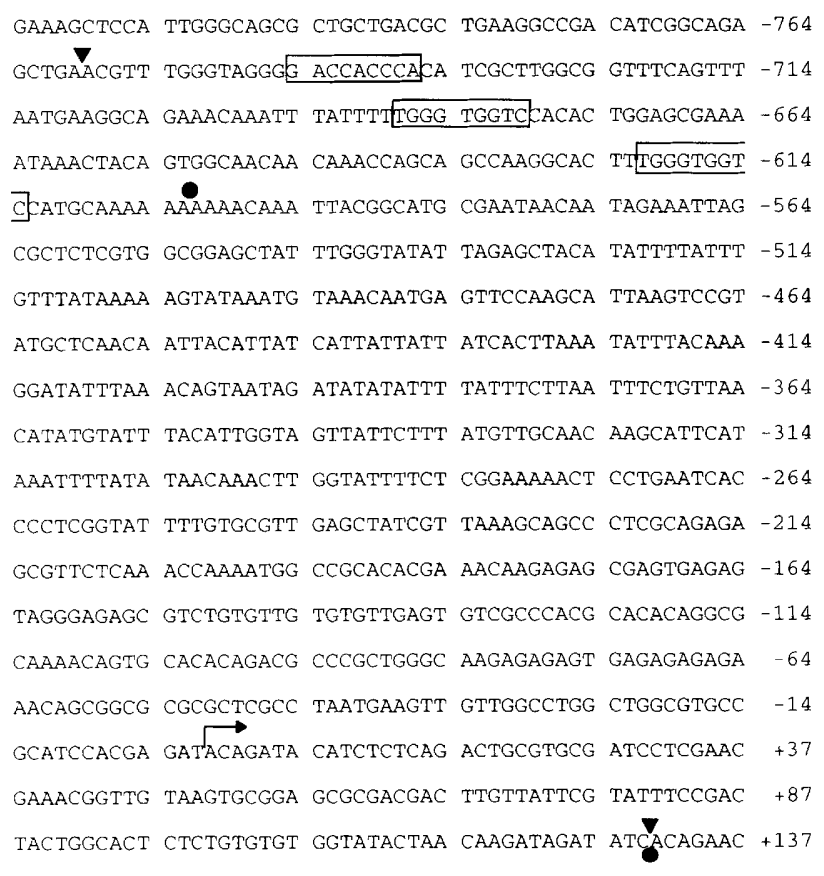

Figure 9. Sequence of the ptc promoter. The predicted transcription start site is indicated (angled arrow). The three GLI consensus-binding sites are indicated by boxes. The two different fragments cloned upstream of the lac $Z$ reporter construct are indicated in solid arrowheads ( $\mathrm{FE}$ construct) or solid circles (GE construct).

within this deleted 156-bp fragment (including the three GLI consensus-binding sites) are necessary for $h h$-dependent transcriptional activation.

\section{Discussion}

Signaling by $h h$ plays a central role in segmental patterning both in the embryo and in the imaginal discs of Drosophila, acting to regulate the transcription of other signal encoding genes, $w g$ or $d p p$, or both, at the anteroposterior compartment boundary. Genetic epistasis analyses have implicated a number of other segment polarity genes in the $h h$ signaling pathway (for review, see Ingham 1995; Perrimon 1995), but among these only one, $c i$, stands out as a possible candidate for the factor that activates directly transcription of target genes in response to $h h$ signaling. The spatial regulation of $c i$ expression is consistent with such a role, both transcript and protein being distributed in a pattern reciprocal to that of $\mathrm{Hh}$ in the embryonic segment and the imaginal discs (Guillen et al. 1995; Johnson et al. 1995; Schwartz et al. 1995). Thus, Ci protein accumulates throughout the anterior compartment such that it is present both in cells that transcribe actively $h h$ target genes as well as in those that do not express these genes but have the potential to do so in response to ectopic $h h$ activity (Ingham 1993; Basler and Struhl 1994). These observations suggest that $\mathrm{Ci}$ is inactive in the majority of cells in which the protein is expressed, its activity being induced only in response to reception of the Hh signal. One way in which 

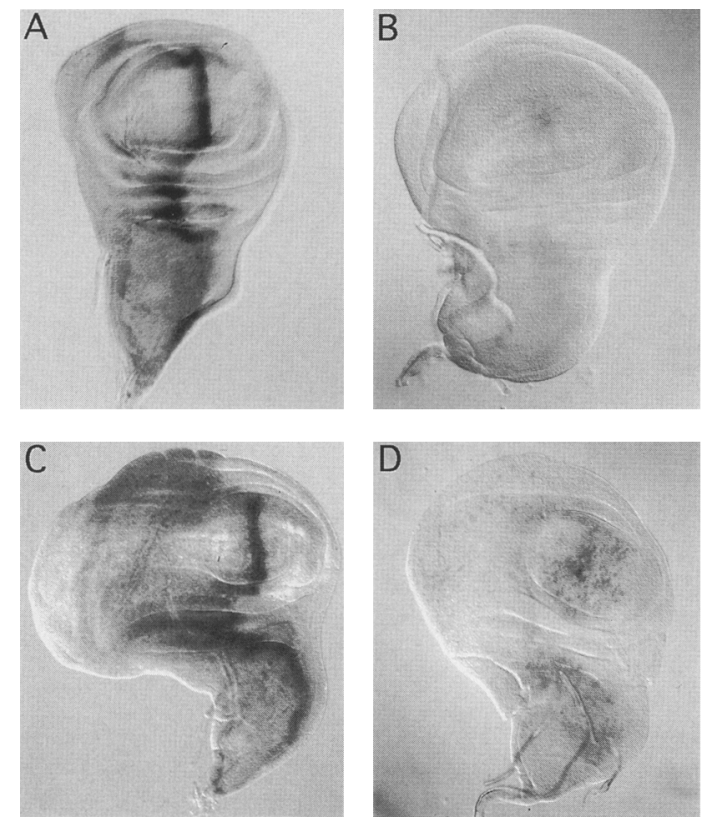

rigure 10. A /58-bp minimal ptc promoter region responds to Shh activity. Ptc-lacZ expression of FE construct containing the three GLI consensus-binding sites $(A)$ and GE deleted from these three sites $(B)$. Note the absence of the compartment boundary when the GLI-binding sites are deleted. (C) Ectopic expression of ptc-lacZ (FE construct) following the overexpression of Shb using the 30A GAL4 enhancer line. $(D)$ In the absence of the GLI-binding sites, the ectopic expression of ptc$l a c Z$ is abolished.

this control might be achieved is by the modulation of protein translation or stability, a possibility suggested by the finding that the levels of $\mathrm{Ci}$ protein are significantly elevated in cells that are actively responding to $\mathrm{Hh}$ (Motzny and Holmgren 1995). Moreover, similarly elevated levels of $\mathrm{Ci}$ are also induced in clones of cells that lack protein kinase A (PKA) activity (Johnson et al. 1995), which itself acts to antagonize the Hh signal response (Jiang and Struhl 1995; Lepage et al. 1995; Li et al. 1995; Pan and Rubin 1995). A possible implication of this latter observation is that PKA acts by modifiying the $\mathrm{Ci}$ protein (either directly or indirectly), causing its inactivation and concomitantly targeting it for degradation. According to this interpretation the increased levels of the protein in $\mathrm{Hh}$ responding cells might be a secondary consequence of the reversal of this modification, leading to increased protein stability that would not in itself be sufficient for the activity of $\mathrm{Ci}$. Although our experiments do not rule out this possibility, they tend to argue against it. Using the GAL4 UAS system we were able to increase artificially the levels of $\mathrm{Ci}$ protein in cells that are not actively responding to $\mathrm{Hh}$ activity. In these experiments, the activity of PKA is unaffected, therefore the exogenous $\mathrm{Ci}$ protein should be subject to any PKA-mediated modification experienced by its endogenous counterpart. Nevertheless, such elevated levels of $\mathrm{Ci}$ were sufficient to activate all of the targets of Hh signaling tested, suggesting that it is the level of $\mathrm{Ci}$ protein that is the principal determinant of its activity. We cannot exclude the possibility, however, that the unusually high levels of $\mathrm{Ci}$ induced in these experiments titrate out the activity of PKA or other negative regulators of $\mathrm{Ci}$ activity.

An interesting and unexpected aspect of our findings is the differential response of the different $\mathrm{Hh}$ target genes to this ectopic Ci activity. Using the GAL4 line 30A to drive expression around the entire wing blade primordium, we find that $\mathrm{Ci}$ activity is sufficient to activate $p t c$ transcription in both anterior and posterior compartments, whereas activation of $d p p$ is restricted precisely to the anterior compartment. This suggests a fundamental difference in the way in which these two genes are normally regulated; previous studies have shown that $d p p$ is under direct negative regulation by En (Sanicola et al. 1995|, and our results indicate that Ci activity is unable to overcome this repression. In contrast, the repression of $p t c$ transcription by en activity, appears to be an indirect consequence of the repression of $c i$ transcription by en (Eaton and Kornberg 1990). Normally this results in the absence of $\mathrm{Ci}$ protein from the posterior compartment, but it is clear from our experiments that provision of Ci protein is sufficient to activate $p t c$ transcription in these cells irrespective of the presence of En protein. It follows that the repression of $c i$ transcription by en could provide the basis for limiting the competence of cells to respond to $h h$ signaling to the anterior compartment. A similar situation is revealed in the embryo using the enGAL4 line to drive ectopic ci expression in the posterior "compartment" of each segment. Here, such expression results in the activation of ptc transcription within enexpressing cells. Significantly, such expression also results in the activation of $w g$ transcription; thus, unlike $d p p$, its signaling counterpart in the wing disc $w g$ appears not to be under the direct control of en. Paradoxically, although $w g$ transcription can be induced in the posterior compartment by ectopic $\mathrm{Ci}$ activity, its induction in the anterior compartment by the same protein is restricted to the so-called $w g$ competent cells. These cells are defined by the expression of the Sloppy paired proteins, transcription factors whose activity is essential for normal wg expression (Cadigan et al. 1994). Neither Slp protein is expressed in the posterior compartment, however, yet $\mathrm{Ci}$ activity appears to be sufficient to activate $w g$ transcription in these cells. This apparent difference in the requirement for slp activity remains to be explained.

Although the correlation between the levels of Ci protein and the activation of $\mathrm{Hh}$ target genes is strong, the precise manner in which Ci might effect such transcriptional activation has remained a contentious issue (Johnson et al. 1995; Motzny and Holmgren 1995). Although $\mathrm{Ci}$ shows strong sequence homology to members of the GLI family of vertebrate transcription factors (Orenic et al. 1990), the protein is barely detectable in the nuclei of either embryonic or imaginal disc cells (Motzny and Holmgren 1995). To address this paradox, we have taken a functional approach, seeking to define the minimal regions of both the $\mathrm{Ci}$ protein and the cis- 
acting regulatory regions of the $h h$ target gene $p t c$, sufficient to mediate its transcriptional activation. The finding that the zinc finger domain of $\mathrm{Ci}$ alone, when fused to the herpes simplex VP16 activation domain, can activate transcription of both $p t c$ and $d p p$ in imaginal discs, indicates that the specificity of $\mathrm{Ci}$ activity is determined by its putative DNA-binding domain. This result, taken with the effects of replacing the putative activation domain of the protein with the En repressor domain, strongly suggests that $\mathrm{Ci}$ acts as a transcription factor. Conclusive evidence for this comes from the demonstration that the intact $\mathrm{Ci}$ protein can activate transcription in yeast from a synthetic promoter containing one consensus-binding site for the vertebrate GLI proteins. These findings, however, cannot distinguish between $\mathrm{Ci}$ regulating the $h h$ target genes directly or regulating the expression of some other transcription factor that itself controls transcription of $p t c, w g$, and $d p p$. Compelling evidence in favor of the former possibility comes from our analysis of the ptc promoter region; by deletion analysis, we have defined a 758-bp ptc upstream regulatory element that directs robust expression along the anteroposterior compartment boundary and is induced by ectopic Shh activity. Deletion of 156 bp containing three consensus GLI-binding sites from this fragment completely abolishes this expression.

Taken together, these results represent the first demonstration that $\mathrm{Ci}$ acts as a transcription factor and provide compelling evidence that the induction of $p t c$ transcription by $h h$ is mediated by a direct interaction between $\mathrm{Ci}$ and sequences upstream of the ptc promoter. Although we have not undertaken an analogous analysis of the $d p p$ and $w g$ regulatory elements, we think it likely that they too will be similarly under the direct control of $\mathrm{Ci}$.

Recent studies have indicated a striking conservation of some elements of the $h h$ signaling pathway between Drosophila and vertebrates. Thus, homologs of $p t c$ have been identified in mouse (Goodrich et al. 1996), chicken (Marigo et al. 1996), and zebrafish (Concordet et al. 1996), which in each case are regulated in response to $h h$ signaling in a manner analogous to their Drosophila counterpart. In addition, several lines of evidence have implicated PKA in signaling by vertebrate $\mathrm{Hh}$ family proteins (Fan et al. 1995; Concordet et al. 1996; Hammerschmidt et al. 1996). Although GLI proteins have yet to be implicated directly in such signaling, their patterns of expression are highly suggestive of their involvement (Hui et al. 1994; Vortkamp et al. 1996. It is a strong prediction of our analysis that the induction of direct targets of Shh activity will be mediated similarly by GLIbinding sites in their cis-acting regulatory regions.

\section{Materials and methods}

Fly stocks

Wild-type flies were obtained from Oregon R strain (Lindsley and Zimm 1992). The different GAL4 lines were kindly provided by U. Hinz (University of Köln, Germany), A. Brand (Wellcome/CRC Institute, Cambridge, UK), and N. Perrimon (Har- vard Medical School, Cambridge, MA). The dpp-lacZ (BS3.0; Blackman et al. 1991), ptc-lac- $Z(\mathrm{H} 84)$, and $h h^{\prime \prime}$ stocks were kindly provided by R. Blackman (University of Illinois, Urbana), C. Goodman (University of California, Berkeley), and C. Nüsslein-Volhard (Max Planck Institut für Entwicklungsbiologie, Tübingen, Germanyl, respectively. The 30Adpp-lacZ and $30 \mathrm{Aptc}-1 \mathrm{acZ}$ recombinants were generated in the laboratory. The $h h$ mutant embryos used in the $h h$ rescue experiment were obtained by crossing UASci; $h h^{\prime \prime}$ females to $h$ GAL $4 h h^{\prime \prime}$ males.

\section{Generation of transgenic fly lines}

The different pUAS.T and ptc-lac $Z$ constructs describe in the plasmid section were microinjected into Drosophila embryos from a $y w^{677223}$ stock using standard procedures. For each case, several independent lines were obtained and analyzed to ensure that the observed effects are independent of insertion site.

\section{Cuticle preparation}

Larvae were collected just before hatching, removed from their vitelline membranes manually and mounted in 1:1 lactic acid/ Hoyer's medium.

\section{Plasmids}

UASci A double-stranded oligonucleotide corresponding to the first 53 nucleotides of the Ci-coding sequence was cloned into pBSKS as an EagI-EcoRI fragment to generate KSci5'. The remaining $c i$ sequence was removed from Cil (kindly provided by R. Holmgren) and cloned as an EcoRI fragment into KSci5' to generate $\mathrm{KS} c i \mathrm{FL}$. The full-length $c i \mathrm{cDNA}$ was then removed from this vector and cloned into pUAS.T as an EagI fragment to generate UASci.

UASci $\Delta 3^{\prime}$ KSciFL was digested with SpeI and SmaI. The SpeI site was filled in with Klenow and the vector was ligated to itself to generate $\mathrm{KS} c i \Delta 3^{\prime}$. This vector contains the $c i$ cDNA with a deletion from amino acids 970-1235 that corresponds to a putative transcriptional activation domain. This cis3' was then cloned in pUAS.T as a EagI-KpnI fragment to generate $\mathrm{UAS} c i \Delta 3^{\prime}$.

UAS.Tci $\triangle E n$ A PCR fragment encoding the transcriptional repression domain of Engrailed (amino acids 228-282) (Han and Manley 1993) was cloned as a BglII-BamHI fragment into

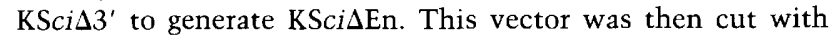
EagI and KpnI and the cisEn insert was cloned into pUAS.T to generate UAS.TcisEn.

UAS.Tci $\Delta 5^{\prime}$ KSciFL was digested with Ncol (position 2184) and EagI $\left(3^{\prime}\right.$ of the coding sequence). The 2325-bp ci fragment was then cloned into KSZFci digested with the same restriction enzymes to generate KScis5'. This vector was then cut with EcoRI and NotI and cloned into pUAS.T to generate UAS.T $c i \Delta 5^{\prime}$

UAS.TZFciVP16 A fragment encoding amino acids 314-609 that includes the entire zinc finger region was generated by PCR, digested by $B g I I I\left(5^{\prime}\right)$ and $B a m H I\left(3^{\prime}\right)$ and then cloned into pKS to generate KSZFci. A BamHI-XbaI PCR fragment encoding the carboxy-terminal transcriptional activation domain of HSV VP16 (amino acids 418-490) was cloned into KSZFci to generate KSZFciVP16. This construct was then cut with EcoRI and NotI and the insert was cloned into pUAS.T to generate UAS.TZFciVP16. 
p601Cibs A double-stranded oligonucleotide corresponding to the GLI consensus-binding site $\left(5^{\prime}\right.$-GACCACCCA-3') was cloned in the yeast vector p601 (Alexandre et al. 1993) at the EcoRI site to generate p601Cibs.

YCGTci The ci-coding sequence (amino acids 18-1262) was cloned in the yeast vector YCGT (Alexandre et al. 1993) to generate YCGTci. The same vector was used to generate YCGTZFciVP16.

ptc-lacZ construct A 888-bp PCR fragment corresponding to the ptc promoter region $(-758$ to +130$)$ was generated and cloned into the wlac- $Z$ vector (Y. Nakano, unpubl.) as a BamHINotI insert to create the FE construct. The same strategy was used to create the GE construct but the 731-bp PCR fragment corresponded to the region -601 to +130 .

\section{Immunocytochemistry}

Wing imaginal discs were dissected in phosphate-buffered saline (PBS) and fixed for $20 \mathrm{~min}$ at $4{ }^{\circ} \mathrm{C}$ in $4 \%$ paraformaldehyde in PBS ( $\mathrm{pH}$ 7.2). Incubation with primary antibodies was carried out overnight at $4^{\circ} \mathrm{C}$ using the following dilutions: monoclonal anti- $\beta$-galactosidase (Promega) at 1:500; rabbit polyclonal anti$\mathrm{Ci}$ at 1:1000 (M. Fietz, unpubl.); rabbit polyclonal anti-Hh (raised against the amino-terminal portion of the protein) (A.M. Taylor, unpubl.) at 1:1000. Double stainings were done in parallel using mouse and rabbit primary antibodies. Confocal images were collected with a Bio-Rad MRC 1000 system and processed with Bio-Rad COMOS and Adobe Photoshop software.

\section{Detection of $\beta$-galactosidase activity}

To detect $\beta$-galactosidase activity in imaginal discs, mature third instar larvae were cut in half in PBS. The anterior halves were inverted and fixed in $4 \%$ glutaraldehyde for $5 \mathrm{~min}$ and washed in PBS. The discs were then stained as described (Ashburner 1989). Stained discs were dissected from the carcass in PBS and mounted in $70 \%$ glycerol for microscopic analysis.

\section{In situ hybridization}

Whole embryos were fixed and hybridized with digoxygeninlabeled single-stranded $w g$ or ptc RNA probes, as described (Ingham et al. 1991\}. For double in situ hybridization, the wg or ptc probes were used with a fluorescein-labeled single-stranded en RNA probe. After the first reaction (using NBT/BCIP) for $w g$ or ptc detection, the embryos were refixed in $4 \%$ formaldehyde and the alkaline phosphatase was inactivated by incubating the embryos overnight in $100 \mathrm{~mm}$ glycine ( $\mathrm{pH} 2.2)$. The next day, the embryos were incubated with the anti-fluorescein AP antibodies for $\mathrm{l} \mathrm{hr}$ and the en staining was then revealed with fast$\mathrm{red} / \mathrm{naphthol}$ in $100 \mathrm{mM}$ Tris $(\mathrm{pH} 8.2)$.

\section{Wing mounting}

Wings from adult flies were dissected in $70 \%$ ethanol, dehydrated, and mounted in Euparal for examination with the compound microscope.

\section{Yeast transformation}

The yeast $S$. cerevisiae strain W303-1A (mata, his3-11,5, trp1-1, ade2-1, leu2-3,112, ura3, ho, can1-100) (kindly provided by Nic Jones, Imperial Cancer Research Fund, London, UK) was trans- formed with the various plasmids using the rapid transformation protocol (Alexandre et al. 1993).

\section{Acknowledgments}

We are grateful to R. Holmgren for supplying the ci cDNA and to U. Hinz, A. Brand, and N. Perrimon for GAL4 enhancer trap lines. We are grateful to Michael Fietz for the polyclonal $\mathrm{Ci}$ anti-serum and the UAS ptc transgenic fly and to Ian Goldsmith for synthesizing oligonucleotides. We would like to thank members of the Ingham laboratory for helpful comments. This research was supported by the Imperial Cancer Research Fund (ICRF) and by a Fellowship from the European Community Human Capital and Mobility Programme to C.A. A.J is in receipt of postgraduate bursaries from Programa Gulbenkian de Doutoramento em Biologia e Medicina and the British Council.

The publication costs of this article were defrayed in part by payment of page charges. This article must therefore be hereby marked "advertisement" in accordance with 18 USC section 1734 solely to indicate this fact.

\section{References}

Alexandre, C., D.A. Grueneberg, and M.Z. Gilman. 1993. Studying heterologous transcription factors in yeast. Methods Companion Methods Enzymol. 5: 147-155.

Ashburner, M. 1989. Drosophila: A laboratory manual. Cold Spring Harbor Laboratory Press, Cold Spring Harbor, NY.

Badiani, P., P. Corbella, D. Kioussis, J. Marvel, and K. Weston. 1994. Dominant interfering alleles define a role for $\mathrm{c}-\mathrm{Myb}$ in T-cell development. Genes \& Dev. 8: 770-782.

Basler, K. and G. Struhl. 1994. Compartment boundaries and the control of Drosophila limb pattern by Hedgehog protein. $\mathrm{Na}$ ture 368: 208-214.

Bender, A. and G.F. Sprague. 1987. Mat $\alpha$ 1, a yeast transcription factor, binds synergistically with a second protein to a set of cell-specific genes. Cell 50: 681-691.

Bitgood, M.J., L. Shen, and A.P. McMahon. 1996. Sertoli cell signaling by Desert hedgehog regulates the male germline. Curr. Biol. 3: 298-304.

Blackman, R.K., M. Sanicola, L.A. Raferty, T. Gillevet, and W.M. Gelbart. 1991. An extensive 3' cis-regulatory region directs the imaginal disc expression of decapentaplegic, a member of the TGF- $\beta$ family in Drosophila. Development 111: 657-665.

Brand, A.H. and N. Perrimon. 1993. Targeted gene expression as a means of altering cell fates and generating dominant phenotypes. Development 118: 401-415.

Cadigan, K.M., U. Grossniklaus, and W.J. Gehring. 1994. Localized expression of sloppy paired protein maintains the polarity of Drosophila parasegments. Genes \& Dev. 8: 899913.

Capdevila, J. and I. Guerrero. 1994. Targetted expression of the signaling molecule decapentaplegic induces pattern duplications and growth alterations in Drosophila wings. EMBO $\%$. 13: $4459-4468$.

Concordet, J.-P., K. Lewis, J. Moore, L.V. Goodrich, R.L. Johnson, M.P. Scott, and P.W. Ingham. 1996. Spatial regulation of a zebrafish patched homologue reflects the roles of sonic hedgehog and protein kinase $\mathrm{A}$ in neural tube and somite patterning. Development 122: 2835-2846.

Couso, J.-P., M. Bate, and A. Martinez-Arias. 1993. A winglessdependent polar coordinate system in Drosophila imaginal discs. Science 259: 484-489. 
Currie, P.D. and P.W. Ingham. 1996. Induction of a specific muscle cell type by a hedgehog-like protein in zebrafish. Nature 382: 452-455.

Eaton, S. and T.B. Kornberg. 1990. Repression of $c i-D$ in posterior compartments of Drosophila by engrailed. Genes \& Dev. 4: 1068-1077

Echelard, Y., D.J. Epstein, B. St.-Jacques, L. Shen, J. Mohler, J.A. McMahon, and A.P. McMahon. 1993. Sonic hedgehog, a member of a family of putative signaling molecules is implicated in the regulation of CNS and limb polarity. Cell 75: 1417-1430.

Fan, C.-M., J.A. Porter, C. Chiang, D.T. Chang, P.A. Beachy, and M. Tessier-Lavigne. 1995. Long-range induction of sclerotome by Sonic hedgehog: A direct role for the amino terminal product of autoproteolytic cleavage and modulation by the cyclic AMP signaling pathway. Cell 81: 457-465.

Fausto-Sterling, A. 1978. Pattern formation in the wing veins of the fused mutant (Drosophila melanogaster). Dev. Biol. 63: $358-369$.

Fietz, M.J., A. Jacinto, A.M. Taylor, C. Alexandre, and P.W. Ingham. 1995. Secretion of the amino-terminal fragment of the Hedgehog protein is necessary and sufficient for hedgehog signaling in Drosophila. Curr. Biol. 5: 643-650.

Forbes, A.J. 1992. "Segment polarity genes in Drosophila development." D.Phil Thesis. University of Oxford, Oxford, UK.

Forbes, A.J., Y. Nakano, A.M. Taylor, and P.W. Ingham. 1993. Genetic analysis of hedgehog signaling in the Drosophila embryo. Development (Suppl.): 115-124.

Goodrich, L.V., R.L. Johnson, L. Milenokovic, J.A. McMahon, and M.P. Scott. 1996. Conservation of the hedgehog/ patched signaling pathway from flies to mice: Induction of a mouse patched gene by hedgehog. Genes \& Dev. 10:310 312.

Grossniklaus, U., R. Kurth-Pearson, and W.J. Gehring. 1992. The Drosophila sloppy paired locus encodes two proteins involved in segmentation that show homology to mammalian transcription factors. Genes \& Dev. 6: 1030-1051.

Guillen, I., J.L. Mullor, J. Capdevilla, E. Sanchez-Herrero, G. Morata, and I. Guerrero. 1995. The function of engrailed and the specification of Drosophila wing pattern. Development 121: $3447-3456$.

Hammerschmidt, M., M.J. Bitgood, and A.P. McMahon. 1996. Protein kinase $\mathrm{A}$ is a common negative regulator of Hedgehog signaling in the vertebrate embryo. Genes \& Dev. 10: $647-658$.

Han, K. and J.L. Manley. 1993. Functional domains of the Drosophila Engrailed protein. EMBO /. 12: 2723-2733.

Hidalgo, A. 1991. Interactions between segment polarity genes and the generation of the segmental pattern in Drosophila. Mech. Dev. 35: 77-87.

Hidalgo, A. and P. Ingham. 1990. Cell patterning in the Drosophila segment: Spatial regulation of the segment polarity gene patched. Development 110: 291-302.

Hooper, J. 1994. Distinct pathways for autocrine and parcrine Wingless signaling in Drosophila embryos. Nature 372: 461-464.

Hui, C.C., D. Slusarski, K.A. Platt, R. Holmgren, and A.L. Joyner. 1994. Expression of three mouse homologs of the Drosophila segment polarity gene cubitus interruptus, Gli, Gli-2, and Gli-3, in ectoderm- and mesoderm-derived tissues suggests multiple roles during postimplantation development. Dev. Biol. 162: 402-413.

Ingham, P.W. 1993. Localised hedgehog activity controls spatially restricted transcription of wingless in the Drosophila embryo. Nature 366: 560-562.

. 1995. Signaling by hedgehog family proteins in Droso- phila and vertebrate development. Curr. Opin. Genet. Dev. 5: 492-498.

Ingham, P.W. and M.J. Fietz. 1995. Quantitative effects of hedgehog and decapentaplegic activity on the patterning of the Drosophila wing. Curr. Biol. 5: 432-441.

Ingham, P.W., K.R. Howard, and D. Ish-Horowicz. 1985. Transcription pattern of the Drosophila segmentation gene hairy. Nature 318: 439-445.

Ingham, P.W., A.M. Taylor, and Y. Nakano. 1991. Role of the Drosophila patched gene in positional signaling. Nature 353: 184-187.

Jiang, J. and G. Struhl. 1995. Protein kinase A and Hedgehog signaling in Drosophila limb development. Cell 80: 563572.

Johnson, R.L., E. Laufer, R.D. Riddle, and C.J. Tabin. 1994. Ectopic expression of Sonic hedgehog alters dorsal-ventral patterning of somites. Cell 79: 1165-1173.

Johnson, R.L., J.K. Grenier, and M.P. Scott. 1995. Patched overexpression alters wing disc size and pattern-transcriptional and posttranscriptional effects on hedgehog targets. Development 121: 4161-4170.

Kinzler, K.W. and B. Vogelstein. 1990. The GLI gene encodes a nuclear protein which binds specific sequences in the human genome. Mol. Cell. Biol. 10: 634-642.

Krauss, S., J.-P. Concordet, and P.W. Ingham. 1993. A functionally conserved homolog of the Drosophila segment polarity gene hedgehog is expressed in tissues with polarizing activity in Zebrafish embryos. Cell 75: 1431-1444.

Lepage, T., S.M. Cohen, F.J. Diaz-Benjumea, and S.M. Parkhurst. 1995. Signal transduction by cAMP-dependent protein kinase A in Drosophila limb patterning. Nature 373: 711-715.

Levin, M., R.L. Johnson, C.D. Stern, M. Kuehn, and C. Tabin. 1995. A molecular pathway determining left-right asymmetry in chick embryogenesis. Cell 82: 803-814.

Li, W., J.T. Ohlmeyer, M.E. Lane, and D. Kalderon. 1995. Function of protein kinase A in Hedgehog signal transduction and Drosophila imaginal disc development. Cell 80: 553-562.

Licht, J.D., M.J. Grossel, J. Figge, and U.M. Hansen. 1990. Drosophila Krüppel protein is a transcriptional repressor. Nature 346: 76-79.

Lindsley, D.L. and G.G. Zimm. 1992. The genome of Drosophila melanogaster. Academic Press, San Diego, CA.

Marigo, V., M.P. Scott, R.L. Johnson, L.V. Goodrich, and C. Tabin. 1996. Conservation in hedgehog signaling: Induction of a chicken patched homolog by Sonic hedgehog in the developing limb. Development 122: 1225-1233.

Mitchell, P.J. and R. Tjian. 1989. Transcriptional regulation in mammalian cells by sequence-specific DNA binding proteins. Science 245: 371-378.

Motzny, C.K. and R. Holmgren. 1995. The Drosophila cubitus interruptus protein and its role in the wingless and hedgehog signal transduction pathways. Mech. Dev. 52: 137-150.

Nellen, D., R. Burke, G. Struhl, and K. Basler. 1996. Direct and long-range action of a DPP morphogen gradient. Cell 85: $357-368$

Nüsslein-Volhard, C. and E. Wieschaus. 1980. Mutations affecting segment number and polarity in Drosophila. Nature 287: 795-801.

Orenic, T., J. Chidsey, and R. Holmgren. 1987. Cell and cubitus interruptus Dominant: Two segment polarity genes on the fourth chromosome in Drosophila. Dev. Biol. 124: 50-56.

Orenic, T.V., D.C. Slusarski, K.L. Kroll, and R.A. Holmgren. 1990. Cloning and characterization of the segment polarity gene cubitus interruptus Dominant of Drosophila. Genes \& Dev. 4: 1053-1067. 
Pan, D. and G.M. Rubin. 1995. cAMP-dependent protein kinase and hedgehog act antagonistically in regulating decapentaplegic transcription in Drosophila imaginal discs. Cell 80: $543-552$.

Pavletich, N.P. and C.O. Pabo. 1993. Crystal structure of a fivefinger GLI-DNA complex: New perspectives on zinc fingers. Science 261: 1701-1707.

Perrimon, N. 1995. Hedgehog and beyond. Cell 80: 517-520.

Riddle, R., R.L. Johnson, E. Laufer, and C. Tabin. 1993. Sonic Hedgehog mediates the polarizing activity of the ZPA. Cell 75: 1401-1416.

Rijsewijk, F., M. Schuermann, E. Wagenaar, P. Parren, D. Weigel, and R. Nusse. 1987. The Drosophila homolog of the mouse mammary oncogene int- 1 is identical to the segment polarity gene wingless. Cell 50: 649-657.

Roberts, D.J., R.L. Johnson, A.C. Burke, C.E. Nelson, B.A. Morgan, and C. Tabin. 1995. Sonic hedgehog is an endodermal signal inducing Bmp-4 and Hox genes during induction and regionalization of the chick hindgut. Development 121: 3163-3174.

Roelink, H., A. Augsburger, J. Heemskerk, V. Korzh, S. Norlin, A. Ruiz i Altaba, Y. Tanabe, M. Placzek, T. Edlund, T.M. Jessell, and J. Dodd. 1994. Floor plate and motor neuron induction by vhh-1, a vertebrate homolog of hedgehog expressed by the notochord. Cell 76: 761-775.

Roelink, H., J. Porter, C. Chiang, Y. Tanabe, D.T. Chang, P.A. Beachy, and T.M. Jessell. 1995. Floor plate and motor neuron induction by different concentrations of the amino terminal cleavage product of Sonic hedgehog autoproteolysis. Cell 81: 445-455.

Ruppert, J.M., B. Vogelstein, K. Arheden, and K.W. Kinzler. 1990. GLI3 encodes a 190-Kilodalton protein with multiple regions of GLI similarity. Mol. Cell. Biol. 10: 5408-5415.

Sanicola, M., J. Sekelsky, S. Elson, and W.M. Gelbart. 1995. Drawing a stripe in Drosophila imaginal discs: Negative regulation of decapentaplegic and patched expression by engrailed. Genetics 139: 745-756.

Schwartz, C., J. Locke, C. Nishida, and T.B. Kornberg. 1995. Analysis of cubitus interruptus regulation in Drosophila embryos and imaginal discs. Development 121: 1625-1635.

Slusarski, D.C., C.K. Motzny, and R. Holmgren. 1995. Mutations that alter the timing and pattern of cubitus interruptus gene expression in Drosophila melanogaster. Genetics 139: 229-240.

Speicher, S.A., U. Thomas, U. Hinz, and E. Knust. 1994. The Serrate locus of Drosophila and its role in morphogenesis of imaginal discs: Control of cell proliferation. Development 120: $535-544$.

Stern, C. and M. Kodani. 1955. Studies on the position effect at the cubitus interruptus locus of Drosophila melanogaster. Genetics 40: 343-373.

Struhl, G. and K. Basler. 1993. Organizing activity of wingless protein in Drosophila. Cell 72: 527-540.

Tabata, T. and T.B. Kornberg. 1994. Hedgehog is a signaling protein with a key role in patterning Drosophila imaginal discs. Cell 76: 89-102.

Thérond, P.P., J.D. Knight, T.B. Kornberg, and J.M. Bishop. 1996. Phosphorylation of the fused protein kinase in response to signaling from hedgehog. Proc. Nat. Acad. Sci. 93:4224-4228.

Triezenberg, S.J., R.C. Kingsbury, and S.L. McKnight. 1988. Functional dissection of VP16, the trans-activator of herpes simplex virus immediate early gene expression. Genes \& Dev. 2: 718-729.

van den Heuvel, M., R. Nusse, P. Johnston, and P.A. Lawrence. 1989. Distribution of the wingless gene product: A protein involved in cell-cell communication. Cell 59: 739-749.
Vortkamp, A., K. Lee, B. Lanske, G.V. Segre, H.M. Kronenberg, and C.J. Tabin. 1996. Indian hedgehog and parathyroid hormone-related protein regulate the rate of cartilage differentiation. Science (in press).

Zarkower, D. and J. Hodgkin. 1992. Molecular analysis of the C. elegans sex-determining gene tra-1: A gene encoding two zinc finger proteins. Cell 70: 237-249.

- 1993. Zinc fingers in sex determination: Only one of the two C. elegans Tra-1 proteins binds DNA in vitro. Nucleic Acids Res. 21: 3691-3698. 


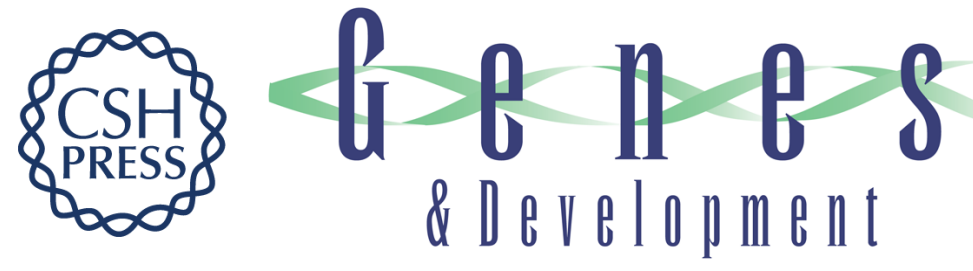

\section{Transcriptional activation of hedgehog target genes in Drosophila is mediated directly by the cubitus interruptus protein, a member of the GLI family of zinc finger DNA-binding proteins.}

C Alexandre, A Jacinto and P W Ingham

Genes Dev. 1996, 10:

Access the most recent version at doi:10.1101/gad.10.16.2003

References This article cites 68 articles, 26 of which can be accessed free at:

http://genesdev.cshlp.org/content/10/16/2003.full.html\#ref-list-1

License

Email Alerting Service

Receive free email alerts when new articles cite this article - sign up in the box at the top right corner of the article or click here.

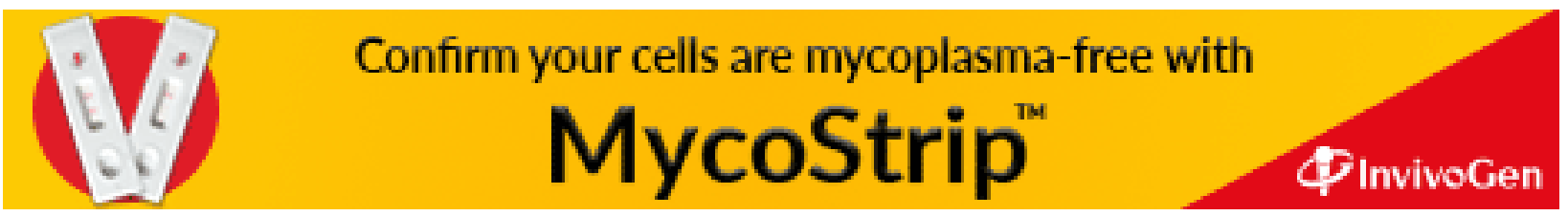

